\title{
Fluorescent analysis reveals that insulin diminishes superoxide increase in neurons induced with toxic glutamate treatment.
}

Vsevolod Pinelis $^{1 *}$, Irina Krasilnikova ${ }^{1}$, Zanda Bakaeva ${ }^{1}$, Alexander Surin ${ }^{1,2}{ }^{6}$, Andrei Fisenko $^{1}$, Dmitry Boyarkin ${ }^{1}$, Olga Krasilnikova ${ }^{3}$, Igor Pomytkin ${ }^{3} 7$ *

${ }^{1}$ National Medical Research Center for Children's Health, Russian Ministry of Health, 102 Lomonosovsky Prospect, Moscow, 119296, Russia

${ }^{2}$ The Institute of General Pathology and Pathophysiology, 8 Baltiyskaya Str., 12 Moscow, 125315, Russia

${ }^{3}$ Department of Advanced Cell Technologies, Sechenov First Moscow State Medical University (Sechenov University), 8 Trubetskaya Str., Moscow, 119991, Russia *Corresponding authors: Vsevolod Pinelis: pinelis@mail.ru; Igor Pomytkin ipomytkin@mail.ru Correspondence: Vsevolod Pinelis National Medical Research Center for Children's Health, Russian Ministry of Health, 2 Lomonosovsky Prospect, Moscow, 119296, Russia. Email: pinelis@mail.ru Phone: +7 916 686-7724

Igor Pomytkin Department of Advanced Cell Technologies, Sechenov First Moscow State Medical 23 University (Sechenov University), 8 Trubetskaya Str., Moscow 119991, Russia. Email: ipomytkin@mail.ru , Phone: +7 495 609-14-00 (3051).

Email addresses:

VP: pinelis@mail.ru

IK: irinakrs181@gmail.com

ZB: bakaeva_z@mail.ru

AS: surin am@mail.ru

AF: direktor@nczd.ru

DB: fly1fly93@yandex.ru

OK: olga4biology@gmail.com

IP: ipomytkin@mail.ru

Conflict of interest: The authors declare that they have no conflict of interest.

Abstract. Glutamate excitotoxicity is implicated in the pathogenesis of many disorders, including stroke, traumatic brain injury, and Alzheimer's disease, for which central insulin resistance is a comorbid condition. Massive glutamate release primarily through ionotropic N-methyl-D-aspartate receptors (NMDARs) causes a sustained rise in $\left[\mathrm{Ca}^{2+}\right]_{\text {i }}$, followed by mitochondrial depolarization and an increase in intracellular $\mathrm{O}_{2}{ }^{*}$

(superoxide) production. Recently, we found that insulin protected neurons against excitotoxicity by diminishing the delayed calcium deregulation (DCD), However, a role of insulin in superoxide production in excitotoxicity still needs to be clarified. The present study is aimed to investigate the effects of insulin on glutamate-evoked superoxide generation and DCD using the fluorescent indicators dihydroethidium, MitoSOX Red, and Fura-FF in rats cultured cortical neurons. We found that insulin significantly diminished both the intracellular and mitochondrial superoxide production in neurons exposed to glutamate and there was a strong linear correlation between $\left[\mathrm{Ca}^{2+}\right]_{\mathrm{i}}$ and intracellular superoxide. MK 801, an inhibitor of NMDAR-gated $\mathrm{Ca}^{2+}$ influx, completely abrogated the glutamate effects in both the presence and absence of insulin. In experiments on sister cultures, insulin diminishes neuronal death. Thus, collectively, 
data obtained suggest that insulin diminishes glutamate-induced superoxide production in neurons via fall of $\left[\mathrm{Ca}^{2+}\right]_{i}$ increased and thereby improves viability of neurons

Keywords: cultured neurons, insulin, glutamate, $\left[\mathrm{Ca}^{2+}\right]_{\mathrm{i}}$, mitochondrial potential, ATP, oxygen consumption rate, superoxide, ROS,

\section{Introduction}

L-Glutamate (glutamate) is an amino acid that serves as the major excitatory neurotransmitter in the central nervous system. Glutamate signalling is necessary for essential brain functions under normal conditions. However, excessive accumulation of extracellular glutamate in a synaptic cleft can trigger a specific pathologic process, known as excitotoxicity, which leads to neuronal cell death. The glutamate excitotoxicity is implicated in the pathogenesis of many disorders, including stroke, traumatic brain injury, and Alzheimer's disease. The glutamate-induced activation of the ionotropic N-methyl-D-aspartate receptor (NMDAR), followed by $\mathrm{Ca}^{2+}$ influx and a biphasic rise in the intracellular free $\mathrm{Ca}^{2+}$ concentration $\left(\left[\mathrm{Ca}^{2+}\right]_{i}\right)$, is considered central to the development of excitotoxicity $[1,2,3,4]$. Experiments with primary neuronal cultures have shown that glutamate exposure causes a rapid, transient increase in $\left[\mathrm{Ca}^{2+}\right]$, followed by a larger secondary $\left[\mathrm{Ca}^{2+}\right]_{i}$ increase. The irreversible larger secondary, $\left[\mathrm{Ca}^{2+}\right]_{\mathrm{i}}$ increase, known as delayed calcium deregulation (DCD), is postulated to be a point-of-no-return in excitotoxicity. Any event occurring downstream of DCD onset is considered to influence the timing of cell death without altering its inevitability [5]. Numerous studies have shown that activation of NMDAR leads to a rise in levels of intracellular superoxide $\left(\mathrm{O}_{2} \bullet^{-}\right)$and non-specified reactive oxygen species (ROS) in neurons. Activation of NMDAR led to a rise non-specified ROS in forebrain neurons [6], cortical neurons [7], striatal neurons [8], and spinal cord neurons [9]. In these studies, mitochondria have been suggested as a major source of NMDAR-induced ROS. An increased production of superoxide upon activation of NMDAR was demonstrated in cultured neurons $[10,11]$ and considered to cause excitotoxic cell death [12].

Mitochondrial aconitase, the tricarboxylic acid cycle (TCA) enzyme, was identified as a principal target of excitotoxic superoxide in cortical cultures [12], which inactivation closely correlated with subsequent neuronal death following excitotoxicity produced by NMDA exposure [13]. Several studies have provided evidence for mitochondria as a source of excitotoxic superoxide production $[14,15]$. However, it still remains unclear how NMDAR-evoked increase of $\left[\mathrm{Ca}^{2+}\right]_{i}$ drives superoxide formation in mitochondria. There is evidence that nicotinamide adenine dinucleotide phosphate (NADPH) oxidase-2 (NOX2), rather than mitochondria, as the primary source of NMDAR-induced superoxide production in cultured cortical and hippocampal neurons $[16,17,18,19]$, as well as in mouse hippocampus in vivo [18]. Interventions that prevent NOX2 activation have been shown to prevent excitotoxic cell death $[16,17]$, indicating that NOX2 is a major source of excitotoxic superoxide. The NMDAR-induced NOX2 activation requires not only an ionotropic $\mathrm{Ca}^{2+}$ influx, but concomitant non-ionotropic NMDAR signalling via the association of phosphoinositide 3-kinase (PI3K) and the GluN2B subunit of NMDAR [20]. NOX2 is a transmembrane enzyme, which functions as a transporter of electrons from cytoplasmic NADPH across membranes by the NOX2 protein complex to reduce molecular oxygen in the extracellular space or in the lumen of intracellular organelles [21]. The membrane-bound NOX2 subunit gp91phox within dendrites was present near the surface membrane, vesicular organelles, and mitochondria [22]. Such subcellular localization of the NOX2 complex in neurons 
indicates that NOX2 in theory may produce superoxide both in extracellular milieu and in the intracellular compartments, including mitochondria. In general, there is the consensus on the importance of NMDAR activation and $\mathrm{Ca}^{2+}$ entry for the accumulation of toxic superoxide in neurons in excitotoxicity. Recently, we have demonstrated that insulin protects cultured cortical neurons from death and preserves neuron functions by diminishing the glutamate-evoked rise in $\left[\mathrm{Ca}^{2+}\right]_{i}$ and preventing the onset of DCD [23]. However, the effects of insulin on glutamate-evoked superoxide generation in neurons and relationship between superoxide, NMDAR, and $\left[\mathrm{Ca}^{2+}\right]_{i}$ have never been studied and still need to be clarified. The present study is aimed to investigate the effects of insulin on glutamate-evoked superoxide generation in cultures of rat cortical neurons, with a focus on the relationship between the dynamics of superoxide production and $\left[\mathrm{Ca}^{2+}\right]_{\mathrm{i}}$ in single neurons to extend our previously published research [23] on the neuroprotective role of insulin during glutamate excitotoxicity.

\section{Materials and methods Materials}

All reagents were obtained from Invitrogen or Sigma-Aldrich (Merck, St. Louis, MO, United States). Cell culture supplies were obtained from Invitrogen (Thermo Fisher Scientific, Waltham, MA, United States).

Primary culture of rat cortical neurons. Experiments with animals were performed in accordance with the ethical principles and regulatory documents recommended by the European Convention for the Protection of Vertebrate Animals used for Experimental and other Scientific Purposes, as well as in accordance with the "Good Laboratory Practice Rules", approved by order of the Ministry of Health of the Russian Federation No. $199 \mathrm{n}$ from 4.01.2016. Primary cultures prepared from the cortex of one- or two-day old Wistar rats as previously described [23]. The rats were anesthetized, decapitated, the skull was opened, the brain was withdraw, and then the cortex was isolated by removing the membranes. The extracted tissues were washed in Hanks' solution without $\mathrm{Ca}^{2+}$ and $\mathrm{Mg}^{2+}$ (Gibco, United States) with $0.04 \% \mathrm{NaHCO} 3$ (HBSS), ground, and placed for 15 $\mathrm{min}$ in a $0.05 \%$ papain solution in medium $(10 \mathrm{mg}$ L-cystein $\mathrm{HCl}, 10 \mathrm{mg}$ BSA, $250 \mathrm{mg}$ glucose with $0.02 \%$ EDTA and $5 \mu 1$ DNAse). After incubation in papain, the altered mixture was washed twice with standard Hanks solution (Gibco, United States) with phenol red, and then with MEM culture medium (Minimal Essential Medium, Gibco, United States). Then the cells were dispersed in fresh MEM medium until a homogeneous suspension was obtained, which was pelleted twice in a centrifuge at 1000 $\mathrm{rpm}$. The precipitated cells were resuspended in an appropriate volume of NBM (Neurobasal Medium, Gibco, USA) supplemented with Supplement B-27 (Gibco, USA), GlutaMax (Gibco, USA), penicillin / streptomycin (Gibco, USA) to a suspension concentration of 106 cells / $\mathrm{ml}$. Then the suspension $(200 \mu \mathrm{l})$ was transferred onto coverslips attached to the wells of $35 \mathrm{~mm}$ plastic Petri dishes (MatTek, USA). The slides were precoated with polyethyleneimine $(10 \mathrm{mg} / \mathrm{ml}, 30 \mathrm{~min}$, PEI not bound to the glass was washed with deionized sterile water $2 \times 1 \mathrm{ml}$ ). One hour later, $1.5 \mathrm{ml}$ of neurobasal medium containing 2\% Supplement B27 and $0.5 \mathrm{mM}$ L-glutamine was added. The cells were kept in an incubator at $37^{\circ} \mathrm{C}, 95 \%$ air $+5 \% \mathrm{CO} 2$, and a relative humidity of $100 \%$. Cytosine arabinose (Ara C, $5 \mu \mathrm{M}$ ) was added to the medium for two or three days to prevent the proliferation of glial cells. Every three days, the cells were fed by replacing $1 / 3$ of the old medium with new medium. For phenotyping of cells cultures were stained with specific primary antibodies to $\beta$-tubulin (PA5-85639) and GFAP proteins (OPA106100) as previously described [19]. Cultures with a percentage of neurons of more than $90 \%$ were used in experiments 10-12 days after plating (10-12 days in culture, DIV). 
Before every experiment, bottoms with the cells and plates were washed ten times out of the B27 supplement with a buffer containing: $(\mathrm{mM}): 135 \mathrm{NaCl}, 5 \mathrm{KCl}, 2 \mathrm{CaCl} 2,1$ $\mathrm{MgCl} 2,20$ HEPES, 5 d-glucose; $\mathrm{pH}$ 7.4. Then, the cells were kept in this buffer for one hour before every experiment. The cells were kept in an incubator $(370 \mathrm{C}, 95 \%$ air $+5 \%$ $\mathrm{CO} 2$, relative humidity 100\%) in NBM. Arabinosine monocytoside (AraC, $5 \mu \mathrm{M}$ ) was added to the incubation medium on the $3 \mathrm{rd}$ day of cultivation to prevent the proliferation of non-neuronal cells. After 24 hours, the AraC medium was replaced with fresh one. Every 3-4 days, a third of the culture medium was replaced with a fresh one. The composition of the NMB culture medium for bark cells (per $100 \mathrm{ml}$ of the finished solution): $1 \mathrm{ml}$ of gutamax (GlutaMax), $2 \mathrm{ml}$ of Supplement B-27, $1 \mathrm{ml}$ of antibiotic / antimycotic (penicillin / streptomycin), $96 \mathrm{ml}$ of NMB solution. The cultures were used 10-12 days after planting (10-12 DVK).

Measurement of $\left[\mathrm{Ca}^{2+}\right]_{i}$ and superoxide. For $\left[\mathrm{Ca}^{2+}\right]_{\mathbf{i}}$ measurements, cortical neurons were loaded with a low affinity $\mathrm{Ca}^{2+}$ indicator, Fura-FF $(2 \mu \mathrm{M})$, in the form of acetoxymethyl esters (in AM-form), for $60 \mathrm{~min}$ at $37^{\circ} \mathrm{C}$ with addition a non-ionic detergent, Pluronic F - 127 (0.02\%; Molecular Probes, USA to facilitate the penetration of the FuraFF into the cells. For simultaneous measurements of $\left[\mathrm{Ca}^{2+}\right]_{i}$ and superoxide, cells were loaded with $1 \mu \mathrm{M}$ of dihydroethidium (HE) fluorescent probe or triphenyl phosphonium linked hydroethidine (Mito-HE) fluorescent probe, a.k.a. MitoSOX Red, for the last $20 \mathrm{~min}$ of the "Fura-FF loading period" in buffer at $37^{\circ} \mathrm{C}$. After loading, the cells were washed with saline (hereinafter: normal saline buffer) of the following composition (in $\mathrm{mM}$ ): $\mathrm{NaCl}-135, \mathrm{KCl}-5, \mathrm{CaCl}_{2}-2, \mathrm{MgCl}_{2}-1$, HEPES - 20, glucose 5 , $\mathrm{pH}$ 7.4. Insulin, at $100 \mathrm{nM}$, was added 5 min prior to $100 \mu \mathrm{M}$ glutamate, in $\mathrm{Mg}^{2+}$ free, $10 \mu \mathrm{M}$ glycine containing medium. To wash Glu, a nominally calcium-free solution was used to exclude any pathways for $\mathrm{Ca}^{2+}$ entry from the buffer into the cytosol in the postglutamate period. To assess the amount of $\mathrm{Ca}^{2+}$ accumulated by mitochondria, mitochondria in the final part of the experiments were depolarized using the FCCP protonophore $(1 \mu \mathrm{M})$. To calibrate the maximum signal of the $\mathrm{Ca}^{2+}$ indicator, ionomycin (Iono, $2.5 \mu \mathrm{g} / \mathrm{ml}$ ) in the presence of $5 \mathrm{mM} \mathrm{Ca}^{2+}$ was added at the end of the experiment. The solutions were changed 3 times, $1 \mathrm{ml}$ each. The cells were incubated on a microscope stage at $24-25^{\circ} \mathrm{C}$ (Olympus IX-71, Japan). The Olympus IX-71 microscope was equipped with a $20 \mathrm{x}$ fluorite objective for measuring the fluorescence of various fluorescent probes. The light source is a Xenon arc lamp $(175 \mathrm{~W})$, the light of which alternately passes through interference filters installed in a computer-controlled wheel, which provides filter change in 50-200 ms (Lambda 10-2 Shutter, Instrument Co., CA, USA). A shutter between the lamp and the wheel with light filters blocks the radiation for the time when the signal is not recorded, preventing photo destruction of the object under study. The dichroic mirror reflects the exciting light towards the objective and transmits the radiation from the sample (colored cell) towards the detector. A lens focuses the excitation light on the sample and collects its fluorescence by directing it through a dichroic mirror to a detector. The fluorescence, before reaching the detector, is also passed through a light filter built into the emission filter wheel, which is controlled synchronously with the excitation wheel. The image was detected using a CCD camera (Charge-coupled device) CoolSNAP HQ2 (Photometrics, USA). The Metafluor computer program (Molecular Devices, USA) made it possible to obtain a digital record of the experiment and process it. The recording signals from cell indicators, images were recorded every $30 \mathrm{~s}$. Fura-FF fluorescence was excited alternately at 340 and $380 \mathrm{~nm}$ and recorded at $525 \mathrm{~nm}$. HE and Mito-HE excitation/emission fluorescence were $565 / 610 \mathrm{~nm}$. Raw fluorescence was normalized to baseline levels prior to stimulus. 
Statistical analysis. All data are presented as the mean \pm standard error of mean (SEM). were performed with the GraphPad Prism software. For comparing the difference between multiple groups, one-way analysis of variance (ANOVA), followed by Tukey's post-test for multiple comparisons was used. For comparing the difference in dynamics between groups, two-way ANOVA with repeated measures, followed by Tukey's or Bonferroni's post-test was used. Pearson's correlation coefficient $r$ was used to assess the correlations between groups. Statistically significant results are marked with asterisks, ${ }^{*} \mathrm{p}<0.05 ; *{ }^{*} \mathrm{p}<0.01 ; * * \mathrm{p}<0.001$; and $* * * \mathrm{p}<0.0001$.

\section{Results}

Primary cultured rat cortical neurons were co-loaded with the fluorescent dye hydroethidine (HE) and the low-affinity fluorescent $\mathrm{Ca}^{2+}$ indicator Fura-FF to simultaneously observe changes in intracellular superoxide and $\left[\mathrm{Ca}^{2+}\right]$ i, respectively. To investigate whether insulin can influence glutamate-evoked changes in $\left[\mathrm{Ca}^{2+}\right]$ i and superoxide production, we treated neurons with $100 \mu \mathrm{M}$ glutamate in the presence and absence of $100 \mathrm{nM}$ insulin and monitored the dynamics of intracellular superoxide concentration and $\left[\mathrm{Ca}^{2+}\right]_{i}$ for 30 minutes (Figure 1). Two-way ANOVA with repeated measures revealed significant primary effects of time and treatment on the rise in both $\left[\mathrm{Ca}^{2+}\right]_{\mathrm{i}}$ (Figure $1 \mathrm{~b} ; F_{40,21080}=254.0, p<0.0001$ and $F_{5,527}=116.6, p<0.0001$, respectively) and superoxide levels (Figure $1 \mathrm{~d} ; F_{40,21080}=519.1, p<0.0001$ and $F_{5,527}=$ $196.2, p<0.0001$, respectively). Tukey's post-test showed that glutamate-treated neurons had significantly higher levels of $\left[\mathrm{Ca}^{2+}\right]$ i (Figure $1 \mathrm{~b}$; from 1 to $30 \mathrm{~min} ; p<$ 0.0001 ) and superoxide (Figure 1d; from 6 to $30 \mathrm{~min} ; p<0.001$ to $p<0.0001$ ), as compared to intact neurons. At 30 minutes after treatment, the mean superoxide level in the glutamate-treated neurons was much higher (Figure 1e; $p<0.0001$ ) compared to that in the intact control neurons. Insulin significantly diminished the glutamate-evoked rise in $\left[\mathrm{Ca}^{2+}\right]_{\mathrm{i}}$ (Figure 1b; from 6 to $15 \mathrm{~min} ; p<0.01$ to $p<0.0001$ ) and superoxide levels (Figure 1c; from 9 to $30 \mathrm{~min} ; p<0.05$ to $p<0.0001$ ). At 30 minutes, superoxide production in neurons treated with insulin and glutamate was 35\% lower (Figure 1e; $p<$ $0.0001)$ than that in neurons treated with glutamate alone.

We used Pearson's test to assess the association between superoxide and $\left[\mathrm{Ca}^{2+}\right]_{\mathrm{i}}$ within each period of 30 minutes of glutamate exposure in the absence or presence of insulin. There was a significant linear correlation between the mean levels of $\left[\mathrm{Ca}^{2+}\right]_{i}$ and intracellular superoxide in neurons treated with glutamate in the absence of insulin (Figure 1f; $r=0.96,95 \%$ confidence interval 0.91-0.97; slope $31.97 \pm 1.84 ; F=$ $301.6, p<0.0001)$ and in the presence of insulin $(r=0.98,95 \%$ confidence interval $0.93-0.98$; slope $21.17 \pm 0.82 ; F=666.1, p<0.0001)$.

To examine whether the rise in intracellular superoxide depends on glutamate-evoked $\mathrm{Ca}^{2+}$ influx through NMDAR, we investigated the effects of MK 801, an inhibitor of the NMDA receptor ion channel, on the dynamics of $\left[\mathrm{Ca}^{2+}\right]_{i}$ and superoxide production in neurons treated with glutamate in the presence or absence of insulin. Two-way ANOVA with repeated measures followed by Tukey's post-test analysis (data of Figure 1b,d) showed that MK 801 completely abrogated the glutamate-evoked rise in $\left[\mathrm{Ca}^{2+}\right]_{\mathrm{i}}$ (Figure $1 \mathrm{e} ; p<0.0001$ ) and superoxide levels (Figure 1e; $p<0.0001$ ). There were no 

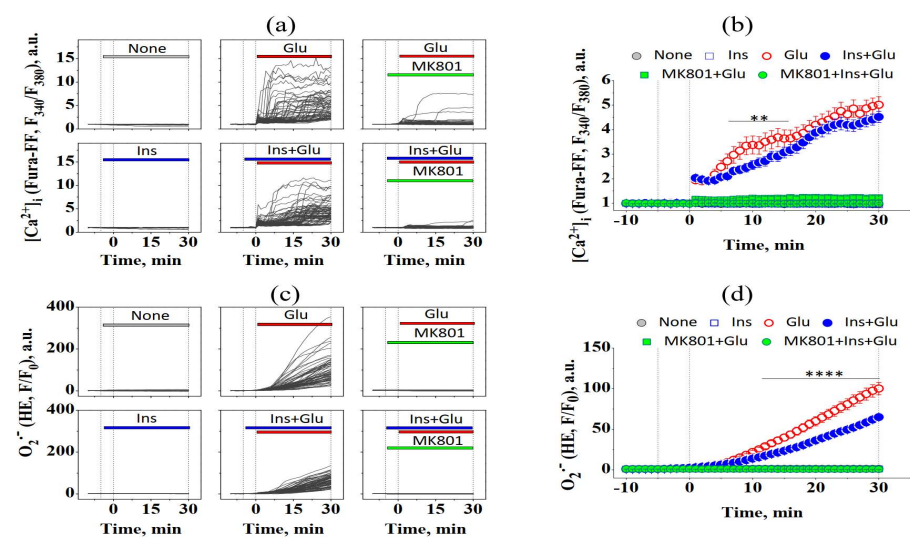

(d)
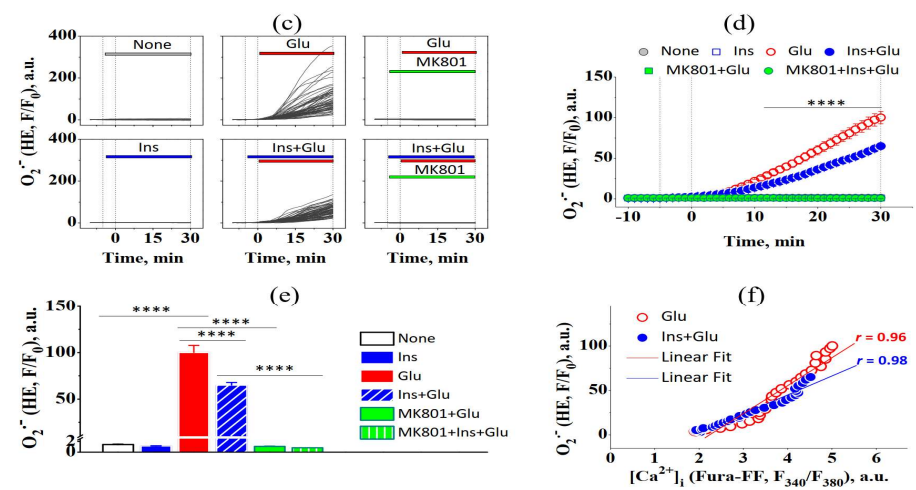

Figure 1 Insulin prevents generation of intracellular superoxide $\left(\mathrm{O}_{2} \bullet^{-}\right)$in rat cortical neurons exposed to glutamate.

(a) The dynamics of Fura-FF fluorescence, a measure of intracellular $\mathrm{Ca}^{2+}\left(\left[\mathrm{Ca}^{2+}\right]\right.$ ), in single neurons treated with none (None; $n=103$ ), $100 \mathrm{nM}$ insulin (Ins; $n=94), 100 \mu \mathrm{M}$ glutamate (Glu; $n=64), 100 \mathrm{nM}$ insulin with $100 \mu \mathrm{M}$ glutamate (Ins+Glu; $n=72$ ), MK 801 with $100 \mu \mathrm{M}$ glutamate (MK801+Glu; $n=100)$, and MK 801 with $100 \mathrm{nM}$ insulin and $100 \mu \mathrm{M}$ glutamate (MK801+Ins $+\mathrm{Glu} ; n=100)$. (b) The dynamics of Fura-FF fluorescence averaged over groups of neurons treated as shown in (a). Data are the mean \pm SEM of the number of neurons, $n .{ }^{* *} p<0.01$ (two-way ANOVA with repeated measures followed by Tukey's post-test) (c) The dynamics of HE fluorescence, a measure of superoxide, in single neurons treated as indicated in (a). (d) The dynamics of HE fluorescence averaged over groups of neurons. Data are the mean $\pm \mathrm{SEM}$ of the number of neurons, $n .{ }^{* * * *} p<0.0001$ (two-way ANOVA with repeated measures followed by Tukey's post-test). (e) The superoxide levels in neurons after 30 minutes of treatment. Data are the mean \pm SEM of the number of neurons, $n . * * * * p<0.0001$ compared to indicated groups (Tukey's post-test). (f) The Pearson's correlations between the means of $\left[\mathrm{Ca}^{2+}\right]_{i}$ and superoxide of Figures $1 \mathrm{~b}, \mathrm{~d}$. The data are the means obtained at every time-point, at 60 -second intervals, within the 30 -minute period of glutamate exposure.

statistically significant differences in $\left[\mathrm{Ca}^{2+}\right]_{\mathrm{i}}$ or superoxide levels between neurons treated with MK 801 and glutamate in the presence $(p>0.05)$ or absence of insulin $(p>$ 0.05 ). These results suggest that NMDAR-gated $\mathrm{Ca}^{2+}$ influx is critically involved in the glutamate-evoked generation of superoxide in cortical neurons exposed to glutamate, independently of the presence or absence of insulin.

To investigate the effects of insulin on glutamate-induced production of mitochondrial superoxide, we used the mitochondria-targeted fluorescent dye Mito-HE (a.k.a.

MitoSOX Red). Rat cortical neurons were co-loaded with Fura-FF and Mito-HE to simultaneously follow changes in intracellular $\left[\mathrm{Ca}^{2+}\right]_{i}$ and mitochondrial superoxide, respectively, and then treated with $100 \mu \mathrm{M}$ glutamate in the presence or absence of 100 $\mathrm{nM}$ insulin. The dynamics of $\left[\mathrm{Ca}^{2+}\right]_{\mathrm{i}}$ and mitochondrial superoxide levels in the single neurons were monitored for 30 minutes (Figures $2 \mathrm{a}$ and $2 \mathrm{c}$, respectively). Two-way 


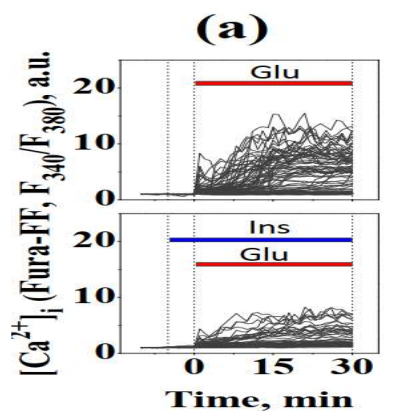

(c)

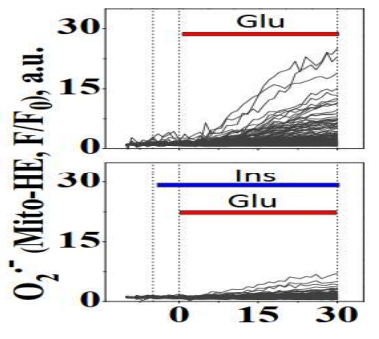

Time, min

(e)

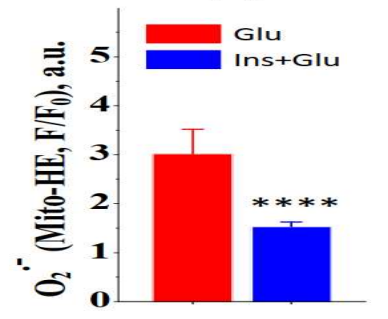

(b)

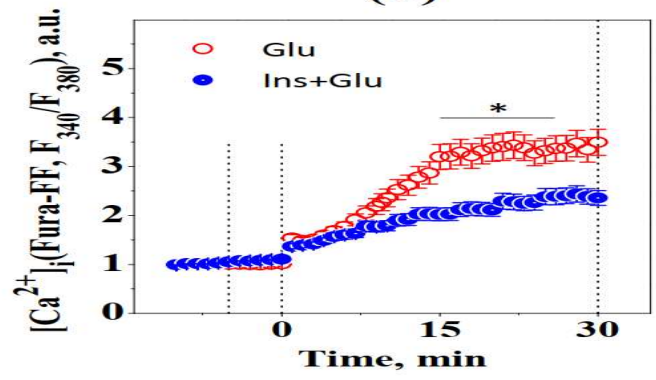

(d)

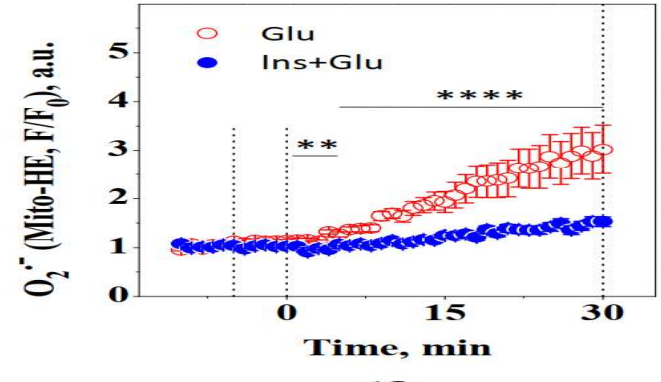

(f)

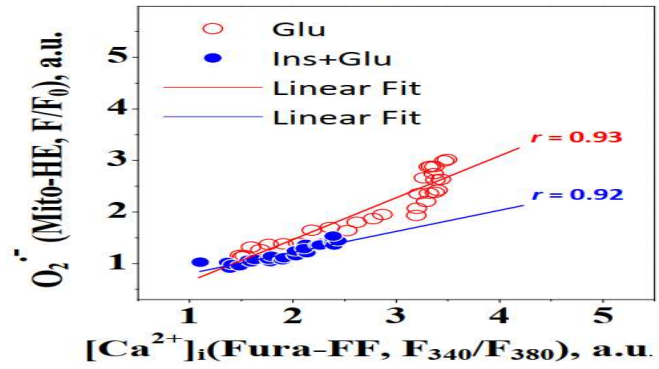

Figure 2 Insulin prevents generation of mitochondrial superoxide $\left(\mathrm{O}_{2}{ }^{-}\right)$in rat cortical neurons exposed to glutamate.

(a) The dynamics of Fura-FF fluorescence, a measure of $\left[\mathrm{Ca}^{2+}\right]$, in single neurons treated with glutamate (Glu; $n=149$ ) or $100 \mathrm{nM}$ insulin and $100 \mu \mathrm{M}$ glutamate (Ins+Glu; $n=95$ ). (b) The dynamics of Fura-FF fluorescence averaged over the groups of neurons treated as shown in (a). Data are the mean \pm SEM of the number of neurons, $n .{ }^{* *} p<0.05$ (two-way ANOVA with repeated measures followed by Bonferroni's posttest) (c) The dynamics of Mito-HE fluorescence, a measure of mitochondrial superoxide, in single neurons treated as indicated in (a). (d) The dynamics of Mito-HE fluorescence averaged over the groups of neurons. Data are the mean \pm SEM of the number of neurons, $n$. ${ }^{*} p<0.01 ; * * * * p<0.0001$ (two-way ANOVA with repeated measures followed by Bonferroni's post-test). (e) The Pearson's correlations between the mean values of $\left[\mathrm{Ca}^{2+}\right]_{i}$ and superoxide levels of Figures $2 \mathrm{~b}, \mathrm{~d}$. The data are the means obtained at every time-point, at 60 -second intervals, within the 30 -minute period of glutamate exposure.

ANOVA with repeated measures revealed significant primary effects of time and insulin on glutamate-induced changes in both $\left[\mathrm{Ca}^{2+}\right]_{\mathrm{i}}$ (Figure $2 \mathrm{~b} ; F_{40,9680}=84.07, p<0.0001$ and $F_{1,242}=7.63, p=0.0062$, respectively) and superoxide levels (Figure $2 \mathrm{~d} ; F_{40,9680}=$ $44.41, p<0.0001$ and $F_{1,242}=22.77, p<0.0001$, respectively). Bonferroni's post-test 
shows that insulin significantly diminished the glutamate-evoked rise in $\left[\mathrm{Ca}^{2+}\right]_{\mathrm{i}}$ (Figure $2 \mathrm{~b}$; from 15 to $24 \mathrm{~min} ; p<0.05$ to $p<0.01$ ) and mitochondrial superoxide levels (Figure $2 \mathrm{~d}$; from 1 to $30 \mathrm{~min} ; p<0.01$ to $p<0.0001$ ). At 30 minutes following treatment, the mean superoxide level in neurons treated with insulin and glutamate was lower by $49 \%$ $(p<0.01)$ compared to that in neurons treated with glutamate alone. There was a significant linear correlation between $\left[\mathrm{Ca}^{2+}\right]_{\mathrm{i}}$ and superoxide levels for neurons treated with glutamate in the absence of insulin (Figure $2 \mathrm{e} ; r=0.93,95 \%$ confidence interval $0.863-0.968$; slope $0.80 \pm 0.06 ; F=188.96, p<0.0001)$ and in the presence of insulin $(r=0.92,95 \%$ confidence interval $0.834-0.961$; slope $0.43 \pm 0.04 ; F=150.8, p<$ $0.0001)$.

Taken together, these results suggest that insulin prevents the glutamate-induced increase in both intracellular and mitochondrial superoxide production via diminishing the NMDAR-dependent rise in $\left[\mathrm{Ca}^{2+}\right]_{\mathrm{i}}$ in neurons.

\section{Discussion}

We previously reported that insulin protects cultured cortical neurons from death and preserves neuron functions by diminishing the glutamate-evoked rise in $\left[\mathrm{Ca}^{2+}\right]_{i}$ and preventing the onset of DCD [23]. Using other methodological approaches, Sh. Maimaiti et al [35] revealed that insulin decreases $\mathrm{Ca} 2+$ current through voltage-gated $\mathrm{Ca}^{2+}$ channels in hippocampal neurons suggesting that insulin restores aging-related changes in calcium regulatory pathways have been shown to lead to higher intracellular calcium levels and an increase in the $\mathrm{Ca}^{2+}$-dependent after hyperpolarization, which is associated with cognitive decline. In present study, we have shown for the first time that insulin decreases the glutamate-evoked generation of both intracellular and mitochondria superoxide, the principal excitotoxic ROS in neurons. The inhibitory effect of insulin on superoxide accumulation is evidently related to its action on $\mathrm{Ca}^{2+}$ dynamics during glutamate exposure, given that insulin suppressed the glutamate-induced rise of $\left[\mathrm{Ca}^{2+}\right] \mathrm{i}$ and there was strong linear correlation between the mean values of $\left[\mathrm{Ca}^{2+}\right]_{i}$ and superoxide levels during the entire period of glutamate exposure in the presence of insulin (Pearson's $r=0.96$ ).

MK 801, the inhibitor of NMDAR-gated $\mathrm{Ca}^{2+}$ influx, completely abrogated the glutamate-induced rise in $\left[\mathrm{Ca}^{2+}\right]_{i}$ and superoxide levels, independently of the presence or absence of insulin, indicating a pivotal role for NMDAR-gated $\mathrm{Ca}^{2+}$ influx in the generation of superoxide in excitotoxicity. These results agree with previous reports indicating the key role of NMDAR-induced $\mathrm{Ca}^{2+}$ influx in superoxide accumulation during excitotoxicity $[6,7,16,17,18,19,20]$.

Antioxidant effects of insulin on the glutamate-induced accumulation of non-specified ROS in differentiated human SH-SY5Y neuroblastoma cells have been shown earlier [24]. However, SH-SY5Y cell line does not appear to be a relevant model for mechanistic studies of oxidative stress in excitotoxicity, given that the glutamateinduced production of ROS in these cells occurs independently of NMDAR activation and $\mathrm{Ca}^{2+}$ influx [25]. The distinctive feature of the present research is that insulin effects were evaluated for the first time on the model of excitotoxicity, in which the production of excitotoxic superoxide in vitro depends critically on the NMDAR-gated $\mathrm{Ca}^{2+}$ influx in the same way as it occurs in vivo [18].

Using fluorescent probes, we found that insulin diminished the glutamate-evoked generation of both intracellular and mitochondria superoxide. HE and Mito-HE fluorescent probes are thought to be appropriate for superoxide measurements in whole cells and mitochondria, respectively [26-28]. Because of its positive charge, Mito-HE preferentially accumulates $\sim 1000$ fold within the mitochondrial matrix [29]. However, 
in excitotoxicity, when mitochondrial depolarization occurs, Mito-HE leaks partially to the cytoplasm [30] and Mito-HE fluorescence may give overestimated values of mitochondrial superoxide. A comparison of fluorescence intensities shows that HE fluorescence increased by 47-fold, while that of Mito-HE increased only 3-fold, after 30 minutes of glutamate exposure. However, a contribution of mitochondrial superoxide production to total superoxide production cannot be quantified from Mito-HE and HE fluorescence data, given that these probes have different rates of oxidation with superoxide [26,27] and their fluorescence yields are influenced by nucleic acid association [30]. Nevertheless, despite its presumably smaller quantities, mitochondrial superoxide may play a critical role in excitotoxic cell death, since inactivation of the tricarboxylic acid cycle enzyme aconitase by mitochondrial superoxide has been shown to be closely correlated with cell death $[12,13]$.

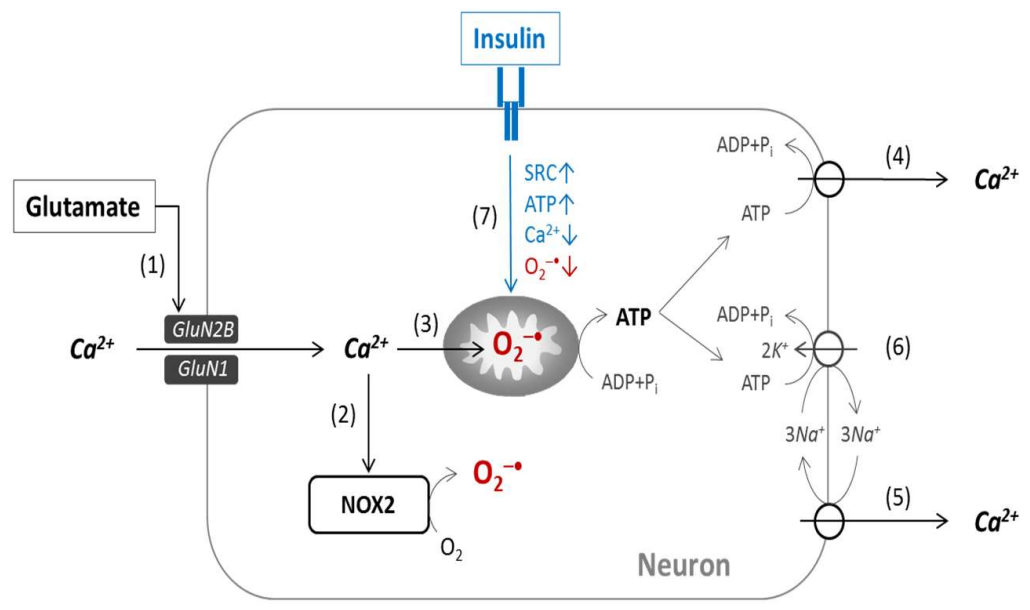

Figure 3. Scheme for functional relationship between NMDAR and insulin receptor (IR) during superoxide generation in excitotoxicity. Following glutamate-evoked NMDAR activation (1), $\mathrm{Ca}^{2+}$ entry leads to a rise in superoxide $\left(\mathrm{O}_{2}^{-}\right)$generation at NOX2 (2) and a rise in superoxide generation in the mitochondrial matrix (3). The NMDAR-gated $\mathrm{Ca}^{2+}$ influx is counterbalanced by ATP-dependent $\mathrm{Ca}^{2+}$ efflux mediated by plasma membrane $\mathrm{Ca}^{2+}$ ATPase (4) and the $\mathrm{Na}+/ \mathrm{Ca}^{2+}$ exchanger (NCX), (5) coupled with $\mathrm{Na}+/ \mathrm{K}+$ ATPase (6). Following $\mathrm{Ca}^{2+}$ entry into the mitochondrial matrix, ATP depletion occurs, thereby affecting ATP-dependent $\mathrm{Ca}^{2+}$ efflux and leading to a rise in $\left[\mathrm{Ca}^{2+}\right]_{\mathrm{i}}$. Insulin significantly increases SRC (7), thereby maintaining ATP production and enhancing ATP-dependent $\mathrm{Ca}^{2+}$ efflux. This prevents $\left[\mathrm{Ca}^{2+}\right]_{i}$ from rising, which in turn lowers superoxide generation at NOX2 and in the mitochondria.

Based on our results from previous research [23] and this study, it is possible to draw a tentative conclusion regarding the mode of action of insulin on superoxide production in excitotoxicity (Figure 3). The major insulin effect on superoxide production relates to preventing the excessive rise in $\left[\mathrm{Ca}^{2+}\right]_{\mathrm{i}}$. In excitotoxic conditions, the NMDAR-gated $\mathrm{Ca}^{2+}$ influx is temporarily counterbalanced with $\mathrm{Ca}^{2+}$ efflux that is governed by plasma membrane $\mathrm{Ca}^{2+}$ ATPase and the $\mathrm{Na}^{+} / \mathrm{Ca}^{2+}$ exchanger (NCX). NCX plays a key role in $\mathrm{Ca}^{2+}$ efflux in excitotoxicity because it has the highest transport capacity for $\mathrm{Ca}^{2+}$ [31,32]. NCX-mediated $\mathrm{Ca}^{2+}$ efflux is ATP-dependent, since $\mathrm{NCX}$ exchanges one $\mathrm{Ca}^{2+}$ for three $\mathrm{Na}^{+}$, and the three $\mathrm{Na}^{+}$are then pumped out by the $\mathrm{Na}^{+} / \mathrm{K}^{+}$ATPase at the expense of one ATP. Prolonged exposure to glutamate leads to ATP depletion and a rise in $\left[\mathrm{Ca}^{2+}\right]_{i}[23,33]$. This rise becomes irreversible when massive $\mathrm{Ca}^{2+}$ influx is no longer 
counterbalanced by $\mathrm{Ca}^{2+}$ efflux, due to NCX switching to the reverse operating mode $\left(\mathrm{NCX}_{r e v}\right)$, which redirects the flow of $\mathrm{Ca}^{2+}$ into the cell $[32,34]$. Thus, maintenance of ATP production is critical for preventing a rise in $\left[\mathrm{Ca}^{2+}\right]_{i}$ in excitotoxicity. In our previous report, we have demonstrated that insulin improved spare respiratory capacity (SRC) in glutamate-treated neurons, thereby inhibiting the glutamate-evoked ATP depletion [23], where SRC relates to the amount of extra ATP that can be produced via oxidative phosphorylation in case of increased energy demand. Therefore, the maintenance of mitochondrial bioenergetics and - primarily - SRC seems to be central to insulin's inhibitory effects on superoxide production during glutamate excitotoxicity.

\section{Conclusion}

We found that insulin prevents glutamate-evoked both intracellular and mitochondrial superoxide generation in primary cultures of rat cortical neurons via diminishing the glutamate-induced rise in $\left[\mathrm{Ca}^{2+}\right]_{\mathrm{i}}$. Given that superoxide causes neuron death, these results seem to explain the neuroprotective action of insulin in glutamate excitotoxicity.

\section{List of abbreviations}

ANOVA: Analysis of variance; $\left[\mathrm{Ca}^{2+}\right]_{\mathrm{i}}$ : Intracellular free $\mathrm{Ca}^{2+}$ concentration; DCD: Delayed calcium deregulation; DIV: Days in vitro; HE: Dihydroethidium; Mito-HE: Triphenyl phosphonium linked hydroethidium; NADPH: Nicotinamide adenine dinucleotide phosphate; NMDAR: N-methyl-D-aspartate receptor; NOX2: NADPH oxidase-2; ROS: Reactive oxygen species.

\section{Ethics approval}

Experiments with animals were performed in accordance with the ethical principles and regulatory documents recommended by the European Convention on the Protection of Vertebrate Animals used for experiments (Guide for the Animals and Eighth Edition. 2010), as well as in accordance with the "Good Laboratory Rules practice", approved by order of the Ministry of Health of the Russian Federation No. 199n of 04/01/2016. The experimental procedures were approved by the Ethics Committee at National Medical Research Center for Children's Health, Russian Ministry of Health.

\section{Consent for publication}

Not applicable

\section{Availability of data and materials}

The datasets, which were used and/or analyzed in the current study, are available from the corresponding author on reasonable request.

\section{Competing interests}

The authors declare that they have no competing interests.

\section{Acknowledgements}

Not applicable

\section{Funding}

This work was supported by Russian Ministry of Health [N. AAAA-A19119012590191-3]. 


\section{Authors' Contributions}

IK, DB, and AS carried out the experimental work. IP and VP wrote the paper. VP, AS, $\mathrm{AF}$, and IP designed and supervised the experiments. VP, IK, HJ, AS, AF, OK, and IP revised the primary manuscript. IK, IP, AS, and VP were responsible for the quality control and analysis. IK, DB, OK, and IP participated in the data analysis. All authors approved the final version of the manuscript.

\section{References}

1. D. G. Nicholls, S. L. Budd, "Mitochondria and neuronal survival," Physiological Reviews, vol. 80, no. 1, pp. 315-360, 2000. doi: 10.1152/physrev.2000.80.1.315.

2. B. Khodorov, "Glutamate-induced deregulation of calcium homeostasis and mitochondrial dysfunction in mammalian central neurons," Progress in Biophysics and Molecular Biology, vol. 86, no. 2, pp. 279-351, 2004. doi: 10.1016/j.pbiomolbio.2003.10.002.

3. T. W. Lai, S. Zhang, Y. T. Wang, "Excitotoxicity and stroke: identifying novel targets for neuroprotection," Progress in Neurobiology, vol. 115, pp. 157-188, 2014. doi: 10.1016/j.pneurobio.2013.11.006.

4. Q. Zhou, M. Sheng, "NMDA receptors in nervous system diseases," Neuropharmacology, vol. 74, pp. 69-75, 2013. doi:10.1016/j.neuropharm.2013.03.030

5. Nicholls DG. Mitochondrial dysfunction and glutamate excitotoxicity studied in primary neuronal cultures. Curr Mol Med. 2004;4:149-177.

6. I. J. Reynolds, T. G. Hastings, "Glutamate induces the production of reactive oxygen species in cultured forebrain neurons following NMDA receptor activation," Journal of Neuroscience, vol. 15, no. 5, pp. 3318-3327, 1995. doi: 10.1523/JNEUROSCI.1505-03318.1995.

7. L.L. Dugan, S. L. Sensi, L. M. Canzoniero et al., "Mitochondrial production of reactive oxygen species in cortical neurons following exposure to N-methyl-Daspartate," Journal of Neuroscience, vol. 15, no. 10, pp. 6377-6388, 1995. doi: 10.1523/JNEUROSCI.15-10-06377.1995.

8. M. Urushitani, T. Nakamizo, R. Inoue et al., "N-methyl-D-aspartate receptormediated mitochondrial $\mathrm{Ca}(2+)$ overload in acute excitotoxic motor neuron death: a mechanism distinct from chronic neurotoxicity after $\mathrm{Ca}(2+)$ influx," Journal of Neuroscience Research, vol. 63, no. 5, pp. 377-387, 2001. doi: 10.1002/10974547(20010301)63:5<377::AID-JNR1032>3.0.CO;2-\%23.

9. Y. Duan, R. A. Gross, S. S. Sheu, "Ca2+-dependent generation of mitochondrial reactive oxygen species serves as a signal for poly(ADP-ribose) polymerase-1 activation during glutamate excitotoxicity," The Journal of Physiology, vol. 585, no. 3, pp. 741-758, 2007. doi: 10.1113/jphysiol.2007.145409.

10. Lafon-Cazal M, Pietri S, Culcasi M, Bockaert J. NMDA-dependent superoxide production and neurotoxicity. Nature. 1993;364:535-7.

11. Lafon-Cazal M, Culcasi M, Gaven F, Pietri S, Bockaert J. Nitric oxide, superoxide and peroxynitrite: putative mediators of NMDA-induced cell death in cerebellar granule cells. Neuropharmacology. 1993; 32:1259-66.

12. Patel M, Day BJ, Crapo JD, Fridovich I, McNamara JO. Requirement for superoxide in excitotoxic cell death. Neuron. 1996; 16:345-55.

13. Li QY, Pedersen C, Day BJ, Patel M. Dependence of excitotoxic neurodegeneration on mitochondrial aconitase inactivation. J Neurochem. 2001 Aug;78(4):746-55. doi: 10.1046/j.1471-4159.2001.00457.x. 
14. Sengpiel B, Preis E, Krieglstein J, Prehn JH. NMDA-induced superoxide production and neurotoxicity in cultured rat hippocampal neurons: role of mitochondria. Eur J Neurosci. 1998 May;10(5):1903-10. doi: 10.1046/j.1460-9568.1998.00202.x.

15. Nguyen D, Alavi MV, Kim KY, Kang T, Scott RT, Noh YH, Lindsey JD, Wissinger B, Ellisman MH, Weinreb RN, Perkins GA, Ju WK. A new vicious cycle involving glutamate excitotoxicity, oxidative stress and mitochondrial dynamics. Cell Death Dis. 2011 Dec 8;2(12):e240. doi: 10.1038/cddis.2011.117.

16. S. W. Suh, E. T. Gum, A. M. Hamby, P. H. Chan, R. A. Swanson, "Hypoglycemic neuronal death is triggered by glucose reperfusion and activation of neuronal NADPH oxidase," The Journal of Clinical Investigation, vol. 117, no. 4, pp. 910918, 2007. doi: 10.1172/JCI30077.

17. H. Girouard, G. Wang, E. F. Gallo et al., "NMDA receptor activation increases free radical production through nitric oxide and NOX2," Journal of Neuroscience, vol. 29, no. 8, pp. 2545-2552, 2009. doi: 10.1523/JNEUROSCI.0133-09.2009.

18. A. M. Brennan, S. W. Suh, S. J. Won et al., "NADPH oxidase is the primary source of superoxide induced by NMDA receptor activation," Nature Neuroscience, vol. 12, no. 7, pp. 857-863, 2009. doi: 10.1038/nn.2334.

19. A. M. Brennan-Minnella, Y. Shen, J. El-Benna, R. A. Swanson, "Phosphoinositide 3-kinase couples NMDA receptors to superoxide release in excitotoxic neuronal death," Cell Death \& Disease, vol. 4, no. 4, pp. e580, 2013. doi: 10.1038/cddis.2013.111.

20. A. M. Minnella, J. X. Zhao, X. Jiang et al., "Excitotoxic superoxide production and neuronal death require both ionotropic and non-ionotropic NMDA receptor signaling," Scientific Reports, vol. 8, no. 1, Article ID 17522, 2018. doi: 10.1038/s41598-018-35725-5

21. Sorce S, Stocker R, Seredenina T, Holmdahl R, Aguzzi A, Chio A, Depaulis A, Heitz F, Olofsson P, Olsson T, Duveau V, Sanoudou D, Skosgater S, Vlahou A, Wasquel D, Krause KH, Jaquet V. NADPH oxidases as drug targets and biomarkers in neurodegenerative diseases: What is the evidence? Free Radic Biol Med. 2017 Nov;112:387-396. doi: 10.1016/j.freeradbiomed.2017.08.006.

22. Glass MJ, Huang J, Oselkin M, Tarsitano MJ, Wang G, Iadecola C, Pickel VM. Subcellular localization of nicotinamide adenine dinucleotide phosphate oxidase subunits in neurons and astroglia of the rat medial nucleus tractus solitarius: relationship with tyrosine hydroxylase immunoreactive neurons. Neuroscience. 2006 Dec 1;143(2):547-64. doi: 10.1016/j.neuroscience.2006.08.051

23. I. Krasil'nikova, A. Surin, E. V. Sorokina et al., "Insulin Protects Cortical Neurons Against Glutamate Excitotoxicity," Frontiers in Neuroscience, vol. 13, pp. 1027, 2019. doi: 10.3389/fnins.2019.0102730

24. M. Nampoothiri, N. D. Reddy, J. John et al., "Insulin blocks glutamate-induced neurotoxicity in differentiated SH-SY5Y neuronal cells," Behavioural Neurology, vol. 2014, Article ID 674164, 2014. doi: 10.1155/2014/674164

25. Z. W. Sun, L. Zhang, S. J. Zhu, W. C. Chen, B. Mei, "Excitotoxicity effects of glutamate on human neuroblastoma SH-SY5Y cells via oxidative damage," Neuroscience Bulletin, vol. 26, no. 1, pp. 8-16, 2010. doi: 10.1007/s12264-0100813-7

26. H. Zhao, S. Kalivendi, H. Zhang et al., "Superoxide reacts with hydroethidine but forms a fluorescent product that is distinctly different from ethidium: potential implications in intracellular fluorescence detection of superoxide," Free Radical Biology and Medicine, vol. 34, no. 11, pp. 1359-1368, 2003. doi: 10.1016/s08915849(03)00142-4 
27. K. M. Robinson, M. S. Janes, M. Pehar et al., "Selective fluorescent imaging of superoxide in vivo using ethidium-based probes," Proceedings of the National Academy of Sciences, vol. 103, no. 41, pp. 15038-15043, 2006. doi: 10.1073/pnas.0601945103

28. J. Chen, S. C. Rogers, M. Kavdia, "Analysis of kinetics of dihydroethidium fluorescence with superoxide using xanthine oxidase and hypoxanthine assay," Annals of Biomedical Engineering, vol. 41, no. 2, pp. 327-337, 2013. doi: 10.1007/s10439-012-0653-x

29. M. Forkink, J. A. Smeitink, R. Brock, P. H. Willems, W. J. Koopman, "Detection and manipulation of mitochondrial reactive oxygen species in mammalian cells," Biochimica et Biophysica Acta (BBA)-bioenergetics, vol. 1797, no. 6-7, pp. 10341044, 2010. doi: 10.1016/j.bbabio.2010.01.022

30. B. M. Polster, D. G. Nicholls, S. X. Ge, B. A. Roelofs, "Use of potentiometric fluorophores in the measurement of mitochondrial reactive oxygen species," Methods in Enzymology, vol. 547, pp. 225-250, 2014. doi: 10.1016/B978-0-12801415-8.00013-8

31. E. Carafoli, L. Santella, D. Branca, M. Brini, "Generation, control, and processing of cellular calcium signals," Critical Reviews in Biochemistry and Molecular Biology, vol. 36, no. 2, pp. 107-260, 2001. doi: 10.1080/20014091074183

32. M. K. Brittain, T. Brustovetsky, P. L. Sheets et al., "Delayed calcium dysregulation in neurons requires both the NMDA receptor and the reverse $\mathrm{Na}+\mathrm{Ca} 2+$ exchanger," Neurobiology of Disease, vol. 46, no. 1, pp. 109-117, 2012. doi: 10.1016/j.nbd.2011.12.051

33. P. S. Brookes, Y. Yoon, J. L. Robotham, M. W. Anders, S.-S. Sheu, "Calcium, ATP, and ROS: a mitochondria love-hate triangle," American Journal of Physiology: Cell Physiology, vol. 287, no. 4, pp. C817-C833, 2004. doi: 10.1152/ajpcell.00139.2004

34. L. Kiedrowski, E. Costa, "Glutamate-induced destabilization of intracellular calcium concentration homeostasis in cultured cerebellar granule cells: role of mitochondria in calcium buffering," Molecular Pharmacology, vol. 47, no. 1, pp. 140-147, 1995. doi: 10.1016/j.nbd.2011.12.051

35. S. Maimaiti, Hilaree Frazier, Katie Anderson, Adam Ghoweri, Lawrence Brewer, Nada Porter, and Olivier Thibault. "Novel calcium-related targets of insulin in hippocampal neurons”. Neuroscience. 2017 November 19; 364: 130-142. doi: $10.1016 /$ j.neuroscience. 2017 